\title{
Editorial
}

Alan N. Sandler MSc MBChB FRCPC, ${ }^{*}$ Joel Katz PhD CPsych $\dagger$

\section{Postoperative analgesia and patient satisfaction}

their health is determined by their own actions (internal MHLC), by powerful other individuals or by chance factors - the latter two dimensions comprising an external MHLC. ${ }^{11,12}$ Effective use of PCA devices requires active, goal-directed behaviour on the part of the patient which may be facilitated by belief's that one's health is in one's own hands. A recent study ${ }^{7}$ found that pain intensity and satisfaction with $i v$-PCA were predicted by health locus of control beliefs. Patients who scored high on the internal MHLC sub scale showed lower pain scores and higher levels of satisfaction. Patients who scored high on the chance MHLC sub scale showed the opposite pattern (i.e., higher pain scores and greater dissatisfaction). These effects were not mediated by total intraoperative dose of opiates, hourly dose of opiates nor by total time of PCA use.

In this issue Egan and Ready have examined the degree of patient satisfaction after the use of either PCA or epidural opioid analgesia for postoperative pain. ${ }^{13}$ To this end they determined which aspects of these two powerful analgesic techniques were seen as advantages or disadvantages by patients using them postoperatively. Satisfaction ratings using a verbal analogue patient satisfaction score $(0=$ "very dissatisfied with pain relief provided", $10=$ "very satisfied with pain relief provided") were assessed as part of the routine care provided by the University of Washington Acute Pain Service (APS). Over a sixmonth period satisfaction scores were collected from 711 patients receiving PCA intravenous morphine and 205 patients receiving epidural morphine after a broad range of surgical procedures. Satisfaction scores were collected on the day that care was terminated by the APS. Both groups reported a high degree of satiisfaction (PCA $=$ $8.6 \pm 1.8$, epidural $=9.0 \pm 1.5$ ).

A random subset of 50 patients each from the epidural and PCA groups was further evaluated on the day the patients began using oral analgesics. Patients in these groups were given a questionnaire listing items which might be expected to contribute to satisfaction or dissatisfaction with the postoperative analgesic method they had used. They were asked to pick three items contributing to their satisfaction or dissatisfaction with the analgesic technique. Patients in the epidural group identified 
the following advantages more often than the PCA group: effective pain relief at rest or while coughing or moving, and having a clear mind. The major disadvantage noted by the epidural group was the high incidence of unpleasant side effects. For the PCA group the greatest problem was the lack of effective analgesia in the post anaesthetic care unit immediately following surgery and before PCA was instituted.

The issue of patient satisfaction is becoming increasingly important as postoperative analgesia delivery systems are left in the hand of the patients. Equally important are the sources of patient satisfaction and dissatisfaction ${ }^{13}$ although ratings of the relative advantages and disadvantages of postoperative analgesic techniques may not always provide clear-cut answers. For example, in the study by Egan and Ready, ${ }^{13}$ rapid onset of PCA is cited as one advantage compared with epidural morphine but pain scores and postoperative opioid dose would be required to interpret this finding. Nevertheless, we suspect that patients in the epidural group never had high pain levels (i.e., did not have to catch up after surgery) since they received epidural local anaesthesia during surgery plus epidural morphine one hour before the end of surgery. We do not have details about the intraoperative management of the PCA group, particularly regarding pre- and intraoperative opioid dosage, but based on one of the important disadvantages reported in the PCA group, it is likely that the PCA group was in more pain than the epidural group immediately after surgery. If this is the case, then what appears to be an advantage for PCA (i.e., works rapidly) may, in reality, be a relative disadvantage since patients who had epidurals would not have required a technique to work rapidly since they were not in as much pain. This possibility is supported by the finding that "pain after surgery before method" was reported as a considerable disadvantage only by patients receiving PCA (i.e., not by patients receiving epidurals). Thus, the apparent advantage of PCA "working quickly" needs to be evaluated in the context of the intense pain these patients endured before their pain was controlled. Egan and Ready's suggestion to start PCA in the recovery room is appropriate but probably won't substitute for a preventive approach such as pre-emptive analgesia ${ }^{14,15}$ plus adequate intraoperative opioid dosage.

This approach used by Egan ${ }^{13}$ and others ${ }^{10}$ represents an important line of research. As Egan and Ready point out, patient satisfaction regarding postoperative analgesia is a complex issue. Progress in this area requires development of well validated and reliable measures of patient satisfaction as well as improved methodology for assessing relative advantages and disadvantages of analgesic regimens. This would include randomized, prospective studies in which measures of postoperative pain and pre-, intra-, and postoperative analgesic dosages are provided. A counterbalanced crossover design may prove helpful in overcoming some of the problems encountered with independent samples when each group rates satisfaction with a different modality. Since patients would have received both forms of treatment, patients would be in a position to rate the advantages and disadvantages of the modalities relative to one another.

Finally, we must be mindful of the potential effects of memory when recalling past experiences of pain. ${ }^{16}$ Ratings of satisfaction were obtained after patients had been taken off PCA or epidural morphine. Many of the sources of patient satisfaction involved judgments of pain or discomfort (and some involved events that occurred three days earlier). Recent work in the field of memory for pain induced by invasive medical procedures shows that retrospective evaluations are strongly correlated with the peak intensity of pain and with the intensity of pain at the end of the procedure but surprisingly, not by the duration of the procedure. ${ }^{17}$ These observations highlight the importance of measuring pain as it is occurring.

In conclusion, collaborative research between anaesthesia and psychology may provide ways of improving postoperative pain management, patient safety, comfort and satisfaction. One potentially fruitful area of research may be to develop empirically-based criteria to match patient characteristics selectively (e.g., psychosocial, psychological, emotional) and postoperative analgesic technique.

\section{L'analgésie postopératoire: le malade est-il satisfait?}

Les morphiniques demeurent la pierre angulaire de l'analgésie postopératoire. Cependant, il a été prouvé que la prescription de morphiniques « à la demande " (PRN) par le personnel soignant était généralement inadéquate pour les besoins de l'analgésie postopératoire. Les faiblesses de cette méthode traditionnelle ont déjà fait l'objet d'une revue par Oden. ${ }^{\prime}$ C'est grâce aux morphiniques rachidiens ${ }^{2}$ et à l'analgésie autocontrôlée (ACP) ${ }^{3}$ que des progrès majeurs ont été réalisés dans le domaine du traitement de la douleur postopératoire. Ordinairement ces techniques ont plus de succès lorsqu'on fait appel à des professionnels de la santé oeuvrant au sein d'un service 
de traitement de la douleur aiguë. ${ }^{4}$ Partout dans le monde, les anesthésistes jouent un rôle déterminant dans l'organisation de ces services. ${ }^{5}$ Vu la diversité et l'efficacité des méthodes disponibles, le choix de la meilleure technique analgésique n'est pas toujours évident. Cette vaste sélection de techniques d'analgésie postopératoire comprend l'administration de morphiniques par rachidienne, par l'ACP (épidural, intraveineuse), la perfusion intraveineuse, la voie orale, sublinguale, rectale, nasale etc.; l'administration d'anesthésiques locaux (rachidiens, régionaux); l'administration d'anti-inflammatoires nonstérïdiens (AINS); l'administration d'inhibiteurs alphaadrénergiques (systémiques, rachidiens); et d'associations de deux ou plusieurs de ces méthodes. ${ }^{6}$

En recherche, la collaboration entre anesthésistes et psychologues peut nous aider à faire le choix de la méthode d'analgésie postopératoire qui convient le mieux à un type de patient. Des études récentes démontrent une relation entre certains facteurs émotionnels et psychologiques et le degré de satisfaction avec l'ACP-i $i$, $^{7-10}$ Une des conclusions les plus intéressantes de ce travail ouvre la voie à l'amélioration du degré de satisfaction et d'efficacité en couplant sélectivement certaines caractéristiques du patient avec celles du système d'administration de l'analgésie. Par exemple, l'échelle multidimensionnelle du point de contrôle de la santé (multidimensional health locus control: MHLC) mesure la conviction avec laquelle un individu croit que ses propres actions influencent son état de santé (MHLC interne) ou que son état de santé est déterminé par d'autres individus puissants ou par le hasard (MHLC externe). ${ }^{11,12}$ L'utilisation efficace d'un module d'ACP nécessite de la part du patient un comportement actif et orienté vers un objectif. $\mathrm{Ce}$ comportement peut être renforcé si le patient est convaincu qu'il contrôle lui-même son état de santé. Une étude récente ${ }^{7}$ montre que l'intensité douloureuse et le degré de satisfaction avec l'ACP-iv pouvent être prédits par le MHLC. Les patients dont la cote de MHLC interne est élevée produisent des scores moins élevés sur l'échelle de la douleur et manifestent un plus grand degré de satisfaction. Par contre, les patients dont la cote est élevée sur la sous-échelle de MHLC de hasard présentent la configuration opposée (scores plus élevés sur l'échelle de la douleur et degré plus faible de satisfaction). La dose peropératoire totale de morphiniques, sa dose horaire et la durée d'utilisation de l'ACP n'ont aucune influence sur ces résultats.

Dans ce numéro du Journal, l'étude d'Egan et Ready examine à la période postopératoire le degré de satisfaction du patient soit avec l'ACP soit les morphiniques épiduraux. ${ }^{13}$ Avec cet objectif, ils ont déterminé quels aspects de ces deux techniques analgésiques efficaces étaient considérés comme avantageux ou désavantageux par les patients qui les utilisaient. Le degré de satisfaction a été évalué sur une échelle verbale analogue $(0=$ insatisfait du soulagement obteno, $10=$ très satisfait du soulagement obtenu) par le service de douleur aiguë de l'université de Washington. Sur une période de six mois, les scores de satisfaction ont été recueillis pour une grande variété d'interventions chez 711 patients sous morphine iv par ACP et 205 patients sous morphine épidurale. Les scores de satisfaction ont été recueillis le jour de l'arrêt du suivi par le service de traitement de la douleur. Les deux groupes ont rapporté un degré élevé de satisfaction (ACP $=8,6 \pm 1,8$, épidurale $=9,0 \pm 1,5$ ).

Deux sous-groupes randomisés de 50 patients chacun pour l'ACP et l'épidurale ont été évalués le jour du passage aux analgésiques oraux. Aux patients de ces groupes, on a remis un questionnaire sur les facteurs contribuant à la satisfaction ou à l'insatisfaction procurée par la technique anesthésique. Les patients du sous-groupe épidurale ont identifiés les avantages suivants plus souvent que les patients du sous-groupe ACP: soulagement efficace de la douleur au repos, à la toux ou à la mobilisation et la clarté d'esprit. Les désavantages majeurs notés par le groupe épidurale concernaient l'incidence élevée d'effets secondaires. Pour le sous-groupe ACP, le problème majeur a consisté au manque d'analgésie efficace à l'unité de soins postanesthésiques immédiatement après la chirurgie, avant la mise en marche de l'ACP.

Depuis la prise en charge par le patient du module d'administration de l'analgésie, la question de la satisfaction du patient devient de plus en plus importante. Les causes de satisfaction et d'insatisfaction ${ }^{13}$ sont toutes aussi importantes malgré le fait que l'interprétation des avantages et désavantages relatifs des techniques analgésiques postopératoires ne puisse pas toujours fournir des réponses définitives. Par exemple, dans l'étude d'Egan et Ready, ${ }^{13}$ l'apparition rapide des effets de l'ACP est considérée comme avantageuse comparativement à la morphine épidurale mais les scores de l'échelle de douleur et les doses de morphiniques postopératoires sont requis pour interpréter ces données. Néanmoins, nous suspectons que les patients du groupe épidurale pourraient avoir eu un degré moins élevé de douleur (donc ils n'avaient pas besoin de rattrapage après la chirurgie) comme ils avaient déjà reçu de l'anesthésie épidurale aux anesthésiques locaux pendant la chirurgie en plus de la morphine épidurale une heure avant la fin de la chirurgie. Nous n'avons pas de détails non plus sur la conduite anesthésique peropératoire utilisée dans le groupe ACP, particulièrement en ce qui a trait aux doses de morphiniques peropératoires. Sur la base de la mention des désavantages importants rapportée par le groupe ACP, il est probable que le groupe ACP était plus souffrant immédiatement après la chirurgie que le groupe épidurale. 
Si tel est le cas, ce qui semble être un avantage de l'ACP (installation rapide de l'analgésie) peut, en réalité, être un désavantage relatif étant donné que les patients sous épidurale n'avait pas besoin d'une technique à début rapide parce qu'ils ne souffraient pas autant. Cette possibilité est confirmée par le fait que la douleur postchirurgicale précédant la mise en marche de la méthode de traitement antalgique n'est considérée comme un désavantage considérable que par les patients sous $\mathrm{ACP}$ et non par les patients sous épidurale. Ainsi, l'avantage apparent (efficacité rapide) de l'ACP doit être evvalué dans le contexte de la perception de la douleur intense qui demande à être soulagée. La suggestion d'Egan et Ready de débuter l'ACP à la salle de réveil est appropriée mais ne peut se substituer à l'analgésie administrée dans un but préventif ${ }^{14,15}$ associée à des dosage de morphiniques adéquats pendant l'intervention.

L'approche utilisée par Egan ${ }^{13}$ et d'autres ${ }^{10}$ ouvre une avenue importante pour la recherche. Comme Egan et Ready le font remarquer, la satisfaction du patient vis à vis de l'analgésie postopératoire est un problème complexe. Pour faire progresser cette recherche, il faudra élaborer des méthodes validées et fiables de mesure du degré de satisfaction aussi bien qu'une meilleure technologie pour évaluer les avantages et désavantages relatifs des méthodes d'analgésie. Ceci signifie, entre autres, des études prospectives randomisées dans lesquelles on effectuera des mesures du degré de douleur pré-, per-, et postopératoires simultanément au dosage des analgésiques. Un plan de chassé-croisé équilibré (counterbalanced crossover design) pourrait s'avérer utile pour surmonter quelques-unes des difficultés causées l'indépendance par des échantillons où chacun des groupes cote le degré de satisfaction obtenu avec chaque méthode de traitement. Comme les patients auraient reçu les deux formes de traitement, ils seraient dans une position de pouvoir coter les avantages et désavantages de chacune des modalités relativement à l'autre.

Finalement nous devons tenir compte des effets possibles de la mémoire sur l'évocation des expériences douloureuses antérieures. ${ }^{16}$ Les cotes de satisfaction ont été recueillies après l'arrêt de l'APC ou de la morphine épidurale. Une bonne partie des renseignements sur la satisfaction des patients comportaient l'appréciation de l'intensité de la douleur ou du manque de confort intéressant quelquefois des événements survenus trois jours auparavant. Une étude récente dans le domaine de la mémoire de la douleur provoquée par des interventions médicales effractives montre que les évaluations rétrospectives sont en étroite corrélation avec l'intensité de pointe de la douleur et avec l'intensité de la douleur de fin dintervention et, curieusement, sans aucune corrélation avec la durée de lintervention. ${ }^{17}$ Cette observation met en relief l'im- portance de mesurer la douleur au moment où elle survient.

Pour conclure, la recherche en collaboration des anesthésistes et des psychologues peut conduire à l'amélioration de la prise en charge de la douleur postopératoire, de la sécurité, du confort et de la satisfaction du patient. Un secteur fertile de recherche pourrait être l'elaboration de critères empiriques assortis sélectivement aux caractéristiques du patient (p. ex. psychosociales, psychologiques, émotionnelles) et à la technique analgésique postopératoire.

\section{References}

1 Oden $R V$. Acute postoperative pain: incidence, severity, and the etiology of inadequate treatment. In: Oden RV (Ed.). Management of Postoperative Pain, Anesthesiology Clinics of North America 1989; 7: 1-17.

2 Cousins MJ, Cherry DA, Gourlay GK. Acute and chronic pain: use of spinal opioids. In: Cousins MJ, Bridenbaugh PO (Eds.). Neural Blockade. Clinical Anesthesia And Management Of Pain. 2nd ed. Philadelphia: Lippincott, 1988; 955.

3 Ferrante MF. Patient-Controlled Analgesia. Boston: Blackwell Scientific Publications, 1990.

4 Ready LB, Wild LM. Organization of an acute pain service: training and manpower. In: Oden RV (Ed.). Management of Postoperative Pain, Anesthesiology Clinics of North America 1989; 7: 229-329.

5 Gould TH, Crosby DL, Harmer M, et al. Policy for controlling pain after surgery: effect of sequential changes in management. BMJ 1992, 305: 1187-93.

6 Dahl JB, Rosenberg J, Dirkes WE, Mogensen T, Kehlet $H$. Prevention of postoperative pain by balanced analgesia. $\mathrm{Br}$ J Anaesth 1990, 64: 518-20.

7 Johnson LR, Magnani B, Chan V, Ferrante FM. Modifiers of patient-controlled analgesia efficacy. Pain 1989; 39: 17-22.

8 Magnani B, Johnson LR, Ferrante MF. Modifiers of patient-controlled analgesia efficacy. II. Chronic Pain. Pain 1989; 39: 23-9.

9 Kluger MT, Owen $H$. Patients' expectations of patientcontrolled analgesia. Anaesthesia 1990; 45: 1072-4.

10 Jamison RN, Tafi K, O'Hara JP, Ferrante FM. Psychosocial and pharmacologic predictors of satisfaction with intravenous patient-controlled analgesia. Anesth Analg 1993: 77: 121-5.

11 Wallston $K$, Wallston B, Kaplan G, Maides $S$. Development and validation of the Health Locus of Control (HCL) Scale. J Consult Clin Psychol 1976; 44: 580-5.

12 Wallston $K$, Wallston $B$, DeVellis $R$. Development of the Multidimensional Health Locus of Control (MHLC) scales. Health Education Monographs 1978; 6: 160-70. 
13 Egan KJ, Ready LB. Patient satisfaction with PCA or epidural morphine. Can J Anaesth 1993; 41: 6-11.

14 Katz J, Kavanagh BP, Sandler AN, et al. Preemptive analgesia: clinical evidence of neuroplasticity contributing to postoperative pain. Anesthesiology 1992; 77: 439-46.

15 Coderre TJ, Katz J, Vaccarino AL, Melzack $R$.

Contribution of central neuroplasticity to pathological pain: review of clinical and experimental evidence. Pain 1993; 52 : 259-85.

16 Erskine A, Morely S, Pierce S. Memory for pain: a review. Pain 1990; 41: 255-65.

17 Redelmeier DA, Kahneman $D$. The pain of invasive procedures: an evaluation of patients' experiences and memories of colonoscopy. Med Decis Making 1992; 12: 338. 